\title{
Melting and crystallization of UHMWPE skived film
}

\author{
Armando Almendarez Camarillo - Stephan Volkher Roth · \\ Peter Bösecke · Stefan Buchner · Klaus Krenn · \\ Rainer Gehrke $\cdot$ Norbert Stribeck
}

Received: 8 March 2006/Accepted: 3 October 2006/Published online: 20 April 2007

(C) Springer Science+Business Media, LLC 2007

\begin{abstract}
Commercial skived film from ultra-high molecular-weight polyethylene (UHMWPE) with considerable uniaxial orientation of lamellae is studied by ultrasmall-angle X-ray scattering (USAXS) and wide-angle Xray scattering (WAXS) during melting and crystallization in order to identify its mechanisms of crystallization. For the analysis of the nanostructure two-dimensional USAXS patterns are analyzed by means of the multidimensional chord distribution function (CDF) method. WAXS shows that crystallization is always isotropic and fast. WAXS reflections are observed before-under certain processing conditions-the SAXS pattern becomes anisotropic. Thus crystallization is decoupled from a slower process of oriented nanostructure formation (nanoforming). If nanoforming is performed isothermally at $105^{\circ} \mathrm{C}$, the evolving nanodomain layers obtain some preferential orientation, as long as the orientation of the melt has not previously been erased by melt-annealing at temperatures of $140{ }^{\circ} \mathrm{C}$ or
\end{abstract}

\author{
A. Almendarez Camarillo $\cdot$ N. Stribeck $(\square)$ \\ Institute of Technical and Macromolecular Chemistry, \\ University of Hamburg, Bundesstr. 45, 20146 Hamburg, \\ Germany \\ e-mail: norbert.stribeck@desy.de \\ S. V. Roth · R. Gehrke \\ HASYLAB at DESY, Notkestr. 85, 22603 Hamburg, Germany \\ P. Bösecke \\ ESRF, 6 rue Jules Horowitz, B.P. 220, Grenoble Cedex 9 38043, \\ France \\ S. Buchner \\ Polymer Consult GmbH, Dorfgrund 6, 22397 Hamburg, \\ Germany \\ K. Krenn \\ Isosport GmbH, Industriestr. 2-8, Eisenstadt 7000, Austria
}

above. Crystallization at temperatures $\geq 110{ }^{\circ} \mathrm{C}$ followed by quenching leads to isotropic nanostructure. Although crystallization is always observed early in the WAXS patterns, the USAXS patterns exhibit only weak discrete scattering during isothermal treatment at temperatures of $110{ }^{\circ} \mathrm{C}$ and higher. At $105{ }^{\circ} \mathrm{C}$ anisotropic isothermal nanoforming starts after $1.5 \mathrm{~min}$. The melting of the original material resembles an inverted random car-parking mechanism. Only next-neighbor correlations are observed among the crystalline layers. The average nanodomain layer thickness is only slightly increasing $(26-30 \mathrm{~nm})$, whereas the long period increase is strong (from $60 \mathrm{~nm}$ to $140 \mathrm{~nm})$.

\section{Introduction}

The mechanisms of structure evolution which are the basis of polymer crystallization have been studied in polymer science for several decades. Since industry has returned to the tailoring of bulk polymer materials, this field is back in the focus of scientific interest [1-8]. As more powerful experimental methods are evolving, the more detailed and profound the understanding of crystallization and nanostructure evolution is becoming.

By means of imaging methods like atomic force microscopy (AFM) or scanning near-field optical microscopy (SNOM) it appears very difficult to in situ monitor a structure transfer process executed under technical conditions with sufficient resolution corresponding to both time and space [9-11]. Utilizing X-ray scattering, such experiments can easily be performed, but the recorded data require model fitting or mathematical evaluation, as long as it 
is not considered to apply simplified notions. Our contribution in this field is based on automatic data evaluation methods for the small-angle X-ray scattering (SAXS) aiming at the model-free visualization of nanostructure and its evolution processes [12-15].

If we combine today sufficiently equipped synchrotron radiation facilities with both an advanced concept of analytics for the investigation of oriented polymer materials, it becomes possible to study processes of structure transfer in two or three dimensions of physical space. Recently we have already reported results on the nanostructure evolution mechanisms studied during melting and crystallization of oriented common polyethylene (Lupolen 6021D) [1620]. Here we report observations that are obtained during similar experiments on a polyethylene material of ultrahigh molecular weight. The studied material is a commercial film with application as the wear layer of skis and snow boards.

\section{Experimental}

Commercial skived film (thickness: $0.76 \mathrm{~mm}$, manufactured by Isosport, Austria) of ultra-high molecular-weight polyethylene (UHMWPE) $\left(M_{w}=4.6 \times 10^{6} \mathrm{~g} / \mathrm{mol}\right)$ is studied. In the differential scanning calorimeter (DSC) the as-received material shows a melting enthalpy of $\Delta H_{m}=179 \mathrm{~J} / \mathrm{g}$, from which a volume crystallinity, $v_{c}=0.58$, is computed using $\Delta H_{m}^{\infty}=239 \mathrm{~J} / \mathrm{g}, \rho_{c}=1.00 \mathrm{~g} /$ $\mathrm{cm}^{3}$ and $\rho_{a}=0.88 \mathrm{~g} / \mathrm{cm}^{3}$ as the melting enthalpy of the crystal, crystalline density, and amorphous density, respectively. The skived film process is applied in order to manufacture films from polymer materials that cannot be extruded (mainly poly(tetra-fluor-ethylene) (PTFE) and UHMWPE). The first production step involves making a cylindrical billet by molding and sintering. In a second step the film material is removed from the rotating billet by use of a blade. In this process isotropic, "semi-directional", and "directional" films can be made.

The material is heated to different melt-annealing temperatures $\left(160,140,138,136,134{ }^{\circ} \mathrm{C}\right)$ in a vacuum and isothermally crystallized at different crystallization temperatures $\left(130,120,110,105,100{ }^{\circ} \mathrm{C}\right)$ while $2 \mathrm{D}$ scattering patterns are taken from time to time. For the interesting constellations found (melting at 140 and $136{ }^{\circ} \mathrm{C}$, crystallization at 110 and $105{ }^{\circ} \mathrm{C}$ ) the processing is thereafter continuously observed in situ by means of time-resolved 2D ultra-small-angle X-ray scattering (USAXS) at HASYLAB, Hamburg, beamline BW4 (X-ray wavelength $0.1366 \mathrm{~nm}$ ). The distance sample-detector is $13,155 \mathrm{~mm}$.

Due to a large beamstop a maximum Bragg spacing of only $270 \mathrm{~nm}$ is resolved. The outer edge of the active area of the detector corresponds to a minimum Bragg spacing of
$22 \mathrm{~nm}$. Thus during our experiments the USAXS beamline at HASYLAB is operated like a very-high-resolution small-angle X-ray scattering (SAXS) camera.

Scattering patterns are recorded by means of a marccd 165 detector (mar research, Norderstedt, Germany) in binned $1024 \times 1024$ pixel mode $(158.3 \mu \mathrm{m}$ quadratic pixel size). Scattering patterns are recorded every $30 \mathrm{~s}$ with an exposure of $28 \mathrm{~s}$.

Environmental data of the experiment are automatically recorded, averaged during exposure, and stored with each pattern for later use. Absorption is measured by means of two ionization chambers. One chamber is positioned directly before the sample, the second chamber is mounted about $30 \mathrm{~cm}$ behind the sample catching SAXS and USAXS. Parasitic background patterns are measured with each synchrotron radiation run.

Additional measurements combining 2D wide-angle X-ray scattering (WAXS) and 2D USAXS during the first 3 min of cooling after melt-annealing at $136{ }^{\circ} \mathrm{C}$ are performed at ESRF, Grenoble, at beamline ID02. Here the exposure is $2 \mathrm{~s}$ for both the USAXS and the SAXS detector. The setup has been described in previous work [20, 21].

Data are evaluated using programs self-written in $p v$-wave [22]. During data evaluation all patterns are normalized with respect to the incident flux. Parasitic background is subtracted under consideration of the measured absorption factor. Blind areas on the detector resulting from the beam stop and its holder are masked. Each pattern is aligned and centered. As far as possible the blind areas are filled utilizing obvious symmetry considerations. This procedure is by no means arbitrary because the measured raw data exhibit perfect 4-quadrant symmetry (fiber symmetry). The success of this procedure is demonstrated by the fact that the resulting pattern is perfectly smooth and no intensity steps are observed at the border between the beam-stop-and-holder region and the region in which valid data have been recorded by the detector.

In order to demonstrate the evolution of anisotropic USAXS during the experiments intensity slices both along the meridian and along the equator are presented, as is frequently done in actual studies. Moreover, examples of the corresponding intensity projections are presented and discussed. As has been deduced several decades ago [13, $23,24]$ such projections but not intensity slices are related to the real-space structure, i.e. the size and arrangement of (in the present study: crystalline) domains in meridional and equatorial direction, respectively.

In order to visualize the domain structure in real space the chord distribution function (CDF) analysis has been utilized [14]. For the CDF analysis the raw USAXS patterns are evaluated. In a first step the remnant tiny central hole is filled by $2 \mathrm{D}$ extrapolation utilizing radial basis 
functions[25]. As described in earlier work [14, 16, 20, 26-32] the scattering intensity is projected onto the $\left(s_{1}, s_{3}\right)$-plane, multiplied by $s^{2}$, then regridded to $512 \times 512$ pixels. A 2 D density fluctuation background is determined (Butterworth low pass filter of 1st order with a cut-off frequency $r_{c}=1$ in units of pixels) and subtracted, resulting in the interference function $G\left(s_{12}, s_{3}\right)$. Background filtering is carried out in two iterated steps for each image. $G\left(s_{12}, s_{3}\right)$ is subjected to $2 \mathrm{D}$ Fourier transformation yielding the 3D chord distribution function (CDF), $z\left(r_{12}, r_{3}\right)$.

The discussion of the CDF in terms of the nanodomain topology $[14,27,28,33]$ inside the sample is straightforward, since the CDF is defined [14] by the Laplacian of Vonk's multidimensional correlation function [34]. As such it presents the autocorrelation of the surfaces of the (nanosize) domains in space in a similar manner as Ruland's interface distribution function (IDF) does [35-37] for 1D structures as a function of distance.

\section{Results and discussion}

Figure 1 shows selected USAXS patterns of the UHMWPE skived film from the series of experiments with a meltannealing temperature of $136{ }^{\circ} \mathrm{C}$. The top row (Fig. 1a) presents the melting of the oriented nanostructure during heating of the material at a rate of $2{ }^{\circ} \mathrm{C} / \mathrm{min}$. A clear twopoint diagram exhibits correlated lamellae extending perpendicular to the machine direction of the film. Increase of the long period as a function of increasing temperature is clearly observed from the scattering patterns. At a temperature of $136{ }^{\circ} \mathrm{C}$ the material is completely molten. Neither wide-angle reflections nor USAXS (cf. Figs. 2, 12) are observed at $136{ }^{\circ} \mathrm{C}$.

Figure $1 \mathrm{~b}$ shows USAXS patterns taken during the isothermal treatment at $110{ }^{\circ} \mathrm{C}$. The scattering patterns are diffuse and almost isotropic. Figure 1c demonstrates that some anisotropy appears during the cooling period after the isothermal phase. Nevertheless, at $30{ }^{\circ} \mathrm{C}$ the material appears almost isotropic.

Isothermal crystallization at $105{ }^{\circ} \mathrm{C}$ is demonstrated in Fig. 1d. Two minutes after quenching to the crystallization temperature the raw scattering pattern is becoming anisotropic with a long-period shoulder, and slightly increasing anisotropy is directly observed in the scattering patterns throughout the isothermal period. Figure 1e shows the ultimate scattering pattern taken at ambient temperature after isothermal treatment at $105{ }^{\circ} \mathrm{C}$ for $20 \mathrm{~min}$. It is differing considerably from the ultimate pattern after crystallization at $110{ }^{\circ} \mathrm{C}$ (Fig. $1 \mathrm{c}$ at $30^{\circ} \mathrm{C}$ ), and shows a clear long period maximum on the meridian. From position and shape of the reflection it is obvious that the nanoscale domains formed lately during quenching are small grains

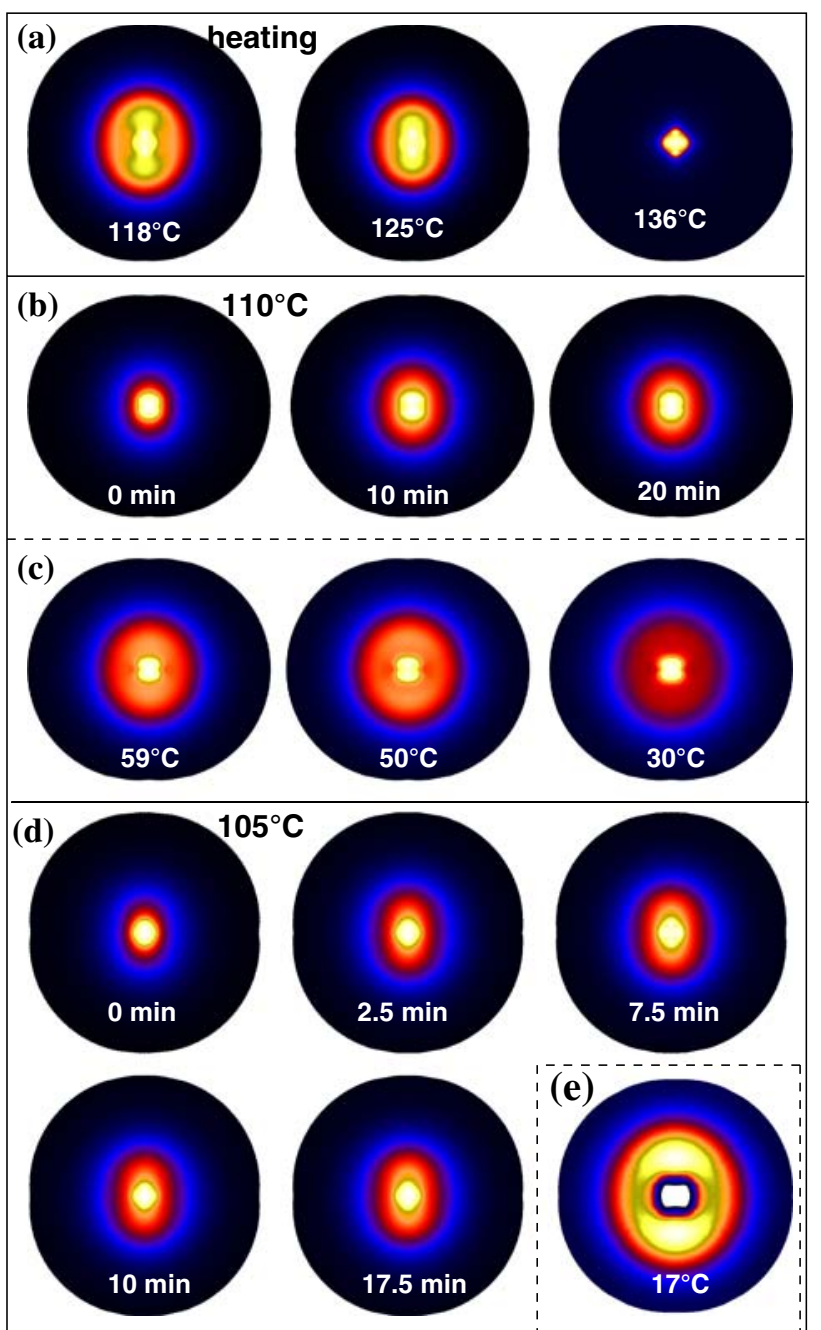

Fig. 1 2D-USAXS of skived film UHMWPE during temperature treatment (HASYLAB, BW4). The temperature is indicated. Fiber axis $s_{3}$ is vertical. Each pattern shows a range $-0.05 \mathrm{~nm}^{-}$ ${ }^{1}<s_{12}, s_{3}<0.05 \mathrm{~nm}^{-1}$ with the scattering vector $\mathbf{s}=\left(s_{12}, s_{3}\right)$, $|\mathbf{s}|=s=\sqrt{s_{12}^{2}+s_{3}^{2}}=(2 / \lambda) \sin \theta .2 \theta$ is the scattering angle. Logarithmic pseudo-color intensity scale. (a) Heating and melting. (b) Isotropic crystallization at $110^{\circ} \mathrm{C}$. (c) Quenching to room temperature after $20 \mathrm{~min}$ at $110{ }^{\circ} \mathrm{C}$. (d) Anisotropic isothermal nanoforming at $105{ }^{\circ} \mathrm{C}$. (e) Ultimate USAXS after treatment at $105{ }^{\circ} \mathrm{C}$

that have been placed in the center of the remnant amorphous regions so that they are fairly well correlated with their neighbors (lamellae). Such structure formation during quenching has previously been identified with other polyethylene material [16].

Figure 2 shows a set of scattering intensity curves $I\left(s_{12}=0, s_{3}\right)$ sliced from the $2 \mathrm{D}$ patterns recorded during heating of the material. The temperature is indicated on the right. Increase of the long period is observed. Nevertheless, the peak itself does not become narrower or more pronounced. Finally, at $136{ }^{\circ} \mathrm{C}$, the SAXS has vanished completely. 


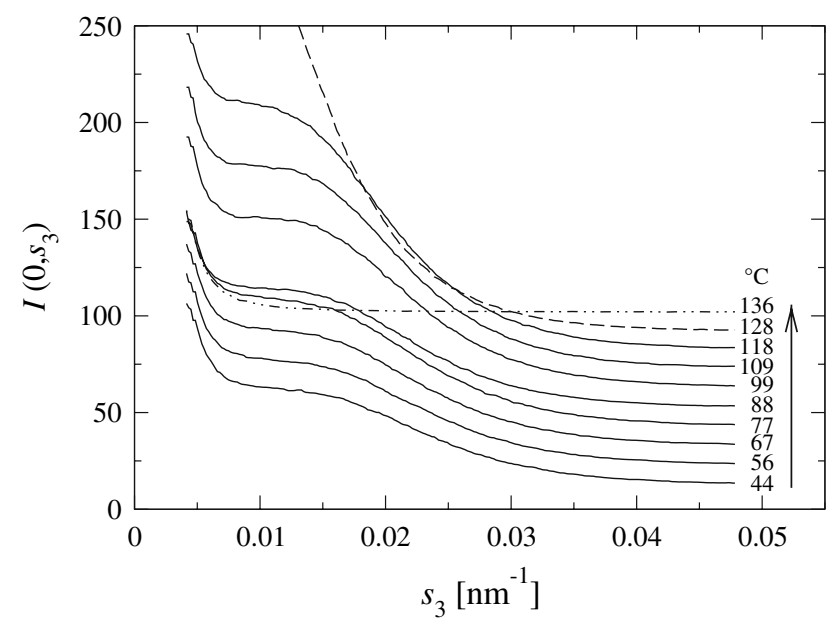

Fig. 2 First heating of UHMWPE skived film. Evolution of the scattering intensity $I\left(s_{12}=0, s_{3}\right)$ along the meridian. Curves are shifted for clarity

The quantitative SAXS intensity changes during isothermal crystallization are shown in the following two figures. Figure 3 shows the data from the isothermal crystallization at $110{ }^{\circ} \mathrm{C}$. The time label indicates the middle of the exposure interval with respect to the begin of quenching. We observe a considerable increase of the SAXS intensity during the first $90 \mathrm{~s}$ both in meridional direction (Fig. 3a) and in equatorial direction (Fig. 3b), but the pattern itself remains diffuse. The sequence of average sample temperatures during exposure of the first four patterns are given in the legend. In all experiments at beamline BW4 the crystallization temperature plateau is reached within less than $60 \mathrm{~s}$ (The heating stage at ID02 is somewhat slower). The first three curves show only diffuse scattering. Beginning with $104 \mathrm{~s}$ after quenching, a weak long period indicates discrete SAXS.

Comparing the intensity slices along the meridian (Fig. 3a) to the slices along the equator (Fig. 3b) we observe only a slight anisotropy. At the lower crystallization temperature of $105{ }^{\circ} \mathrm{C}$ anisotropy of the discrete SAXS becomes considerably higher (Fig. 4). With respect to crystallization at $110{ }^{\circ} \mathrm{C}$ the increase of anisotropy is mainly an effect of a narrower intensity distribution in equatorial direction. Such a narrowing can be explained by agglomeration of a few crystallites into a layer [38, 39], causing liquid scattering (cf. also Fig. 13). As is observed from the scattering curves, the variation of the scattering power

$Q=2 \pi \iint s_{12} I\left(s_{12}, s_{3}\right) \mathrm{d} s_{12} \mathrm{~d} s_{3}$

appears to be nearly identical for the two series. Because of the fact that the SAXS patterns are recorded using a 2D
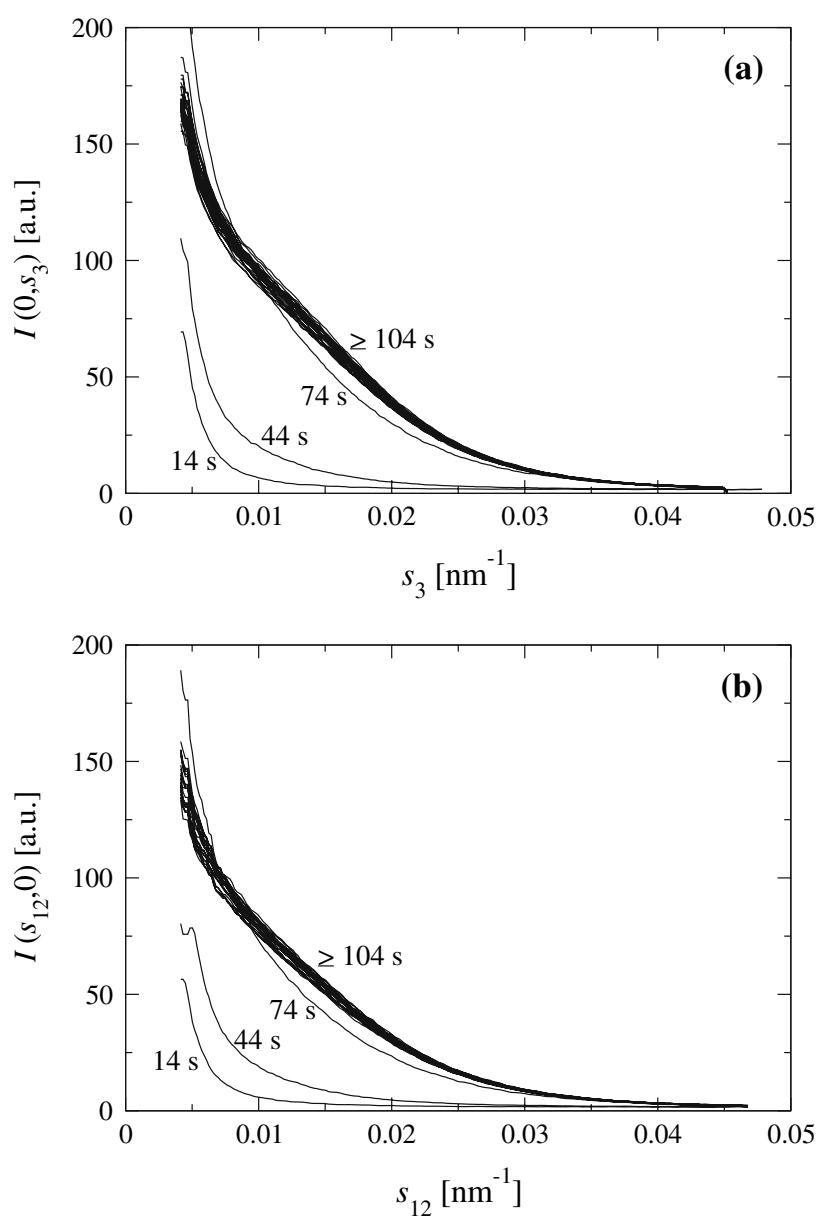

Fig. 3 Isothermal crystallization of UHMWPE. Evolution of the scattering intensity $I$ as a function of time after quenching from $136{ }^{\circ} \mathrm{C}$ to $110{ }^{\circ} \mathrm{C}$. (a) Along the meridian $s_{3}$. (b) Along the equator $s_{12}$. Intensities are normalized with respect to incident flux and sample thickness. Average sample temperatures related to the first four snapshots are $132,118,109,110^{\circ} \mathrm{C}$

detector, $Q$ is readily computed by integration (Fig. 5). The increase of the scattering power during quenching is determined by the cooling rate of the heating stage and thus almost identical in both series. The sample crystallized at $105{ }^{\circ} \mathrm{C}$ shows an almost flat isothermal plateau. Some more pronounced fluctuations of $Q$ are observed in the plateau of the material crystallized at $110^{\circ} \mathrm{C}$.

As here we present intensity slices cut in different directions of the scattering pattern, we are following present common practice in polymer science. Nevertheless, as has been deduced from scattering theory at the end of the 1960s, [23, 24] such slices of scattering intensity do not contain the information on size and arrangement of domains in the slice direction that is of interest to the materials scientists. Instead, they describe the statistics of domain sizes and distances in a projection on to the chosen slicing direction (Fig. 6). Based on this insight mathematicians and engineers working in the field of tomography 

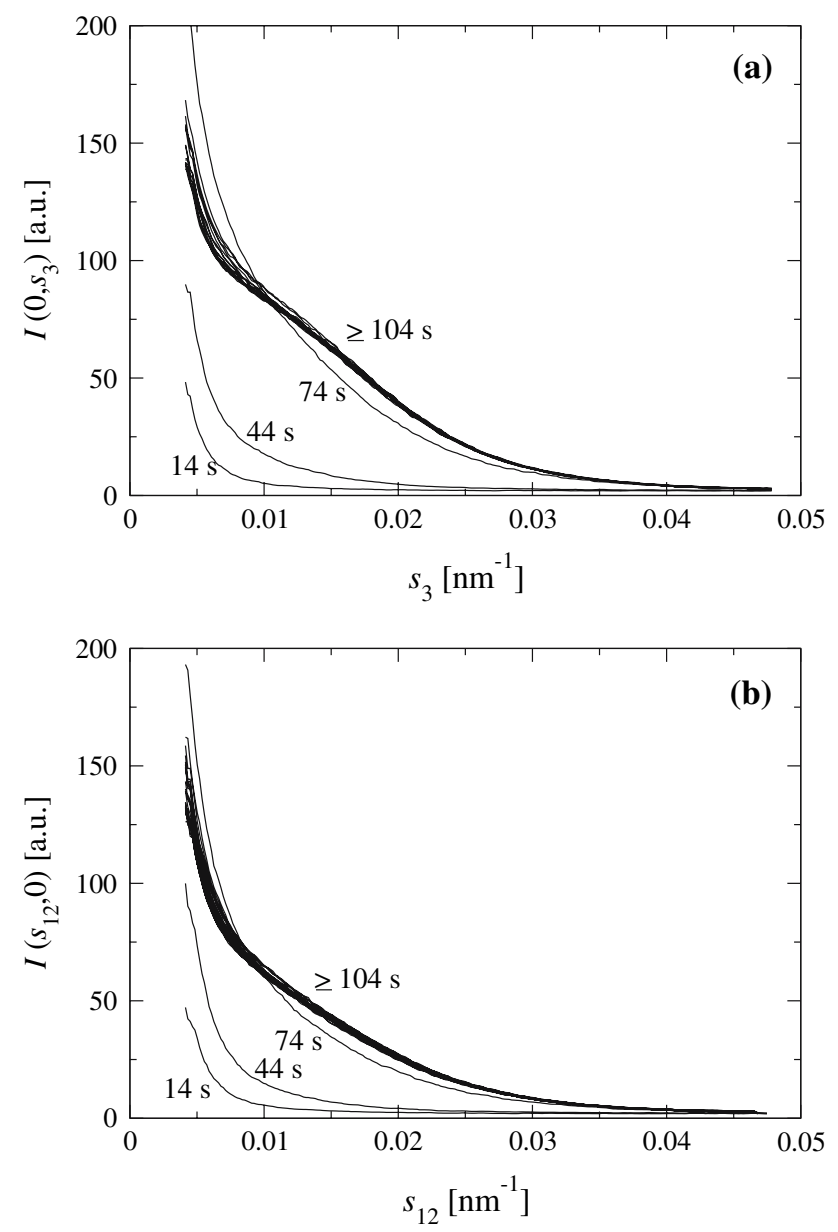

Fig. 4 Isothermal crystallization of UHMWPE. Evolution of the scattering intensity $I$ as a function of time after quenching from $136{ }^{\circ} \mathrm{C}$ to $105{ }^{\circ} \mathrm{C}$. (a) Along the meridian $s_{3}$. (b) Along the equator $s_{12}$. Intensities are normalized with respect to incident flux and sample thickness. Average sample temperatures related to the first four snapshots are $132,116,104,105^{\circ} \mathrm{C}$

have consequently and successfully implemented modelfree reconstruction methods for the imaging of structure during the past decades. In polymer science, on the other hand, it has become widely accepted to first plot intensity slices in a double-logarithmic plot, and, second to discuss the observed features based on notions that are only valid under restricting assumptions. Concerning the progress of SAXS method this development appears to be a dead end. On the other hand, the power of to-date tomographic methods indicates that a real-space-analysis method of short-range correlated structure has some potential for scientific progress.

Figure 6 shows a sketch of a distorted domain structure with uniaxial preferential orientation. According to Bonart [23] the "longitudinal structure" in the sample is the sequence of domain thicknesses and distances measured in fiber direction. In the sketch we have indicated some of the corresponding "section long periods" by black, horizontal

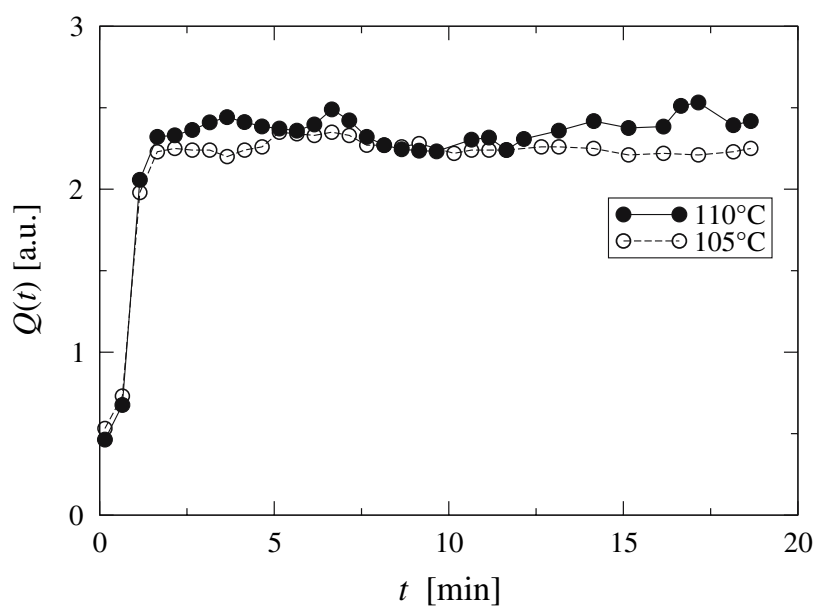

Fig. 5 Variation of the scattering power or invariant, $Q$, during isothermal crystallization of UHMWPE skived film at different temperatures

lines. As a result of the Fourier slice theorem the information on this structural feature is contained in the projection of the scattering intensity onto the fiber axis [23, 24]. On the other hand, the popular intensity sections (in the example: "in fiber direction") contain information on the section long period plus the projections of all "out-of direction" long periods on the (example) fiber direction. In the sketch some of the corresponding additional long periods are indicated by gray, bold horizontal lines. From this mathematical and geometrical relation it can be concluded that-if the correlation among domains is strongly decreasing as a function of their distance from each other in fiber direction - the intensity section may exhibit a long

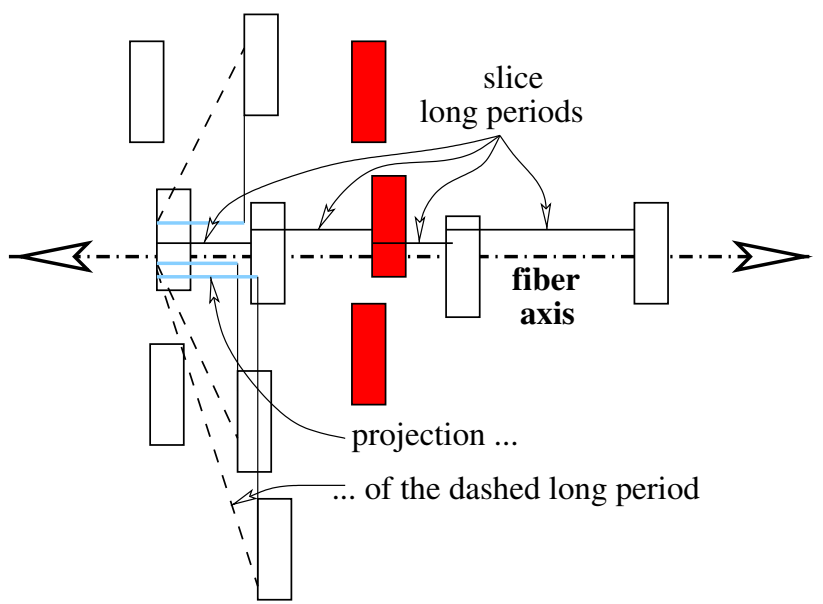

Fig. 6 Sketch of a distorted domain structure explaining the different information content of SAXS intensity slices and SAXS intensity projections. SAXS intensity projections inform on the interesting slice long periods. Additionally, SAXS intensity sections average over all projections of "out-of-direction"' long periods in the material. The domains filled in dark color indicate a subset of domains with lateral correlation (block structure) 
period peak that is almost invisible in the intensity projection. This happens if the distribution of projected long periods is narrower than the distribution of slice long periods.

Figure 7 shows the evolution of the projected SAXS intensities during quenching and isothermal crystallization of the UHMWPE skived film. As compared to the corresponding sections here only a faint indication of discrete SAXS is observed. Thus the evolution of domain anisotropy and its relation to the already addressed liquid scattering appears much clearer in this plot that is based on proper application of scattering theory.

Analysis of the recorded data in real space has been performed by means of the multidimensional CDF [14]. One of the resulting CDFs, $z(\mathbf{r})$, and its features is presented in Fig. 8. The left plot in the figure presents a view of the CDF. The central elevation may be considered a double triangle that is subjected to some disorientation
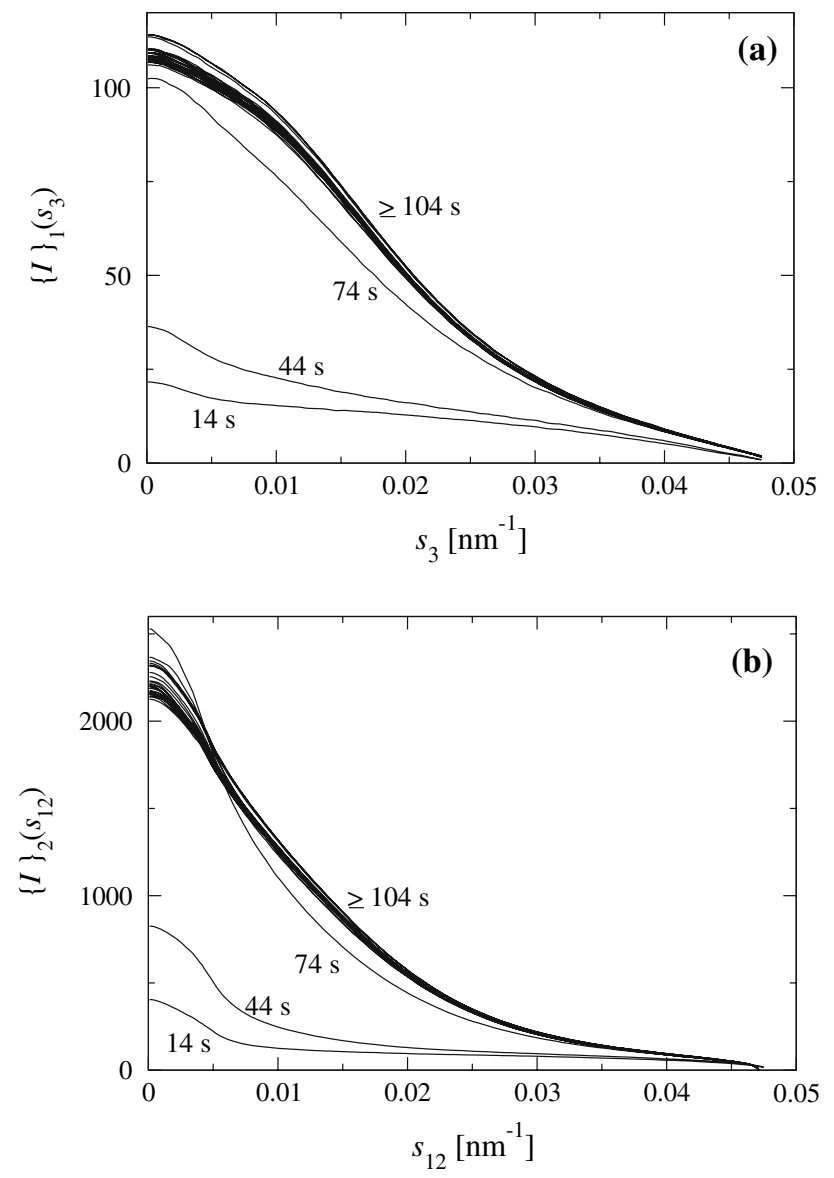

Fig. 7 Isothermal crystallization of UHMWPE. Evolution of the projected scattering intensity $\{I\}$ as a function of time after quenching from $136{ }^{\circ} \mathrm{C}$ to $105{ }^{\circ} \mathrm{C}$. (a) Projected to the meridional direction $s_{3}$. (b) Projected to the equatorial plane $s_{12}$. Intensities are normalized with respect to incident flux and sample thickness. Average sample temperatures related to the first four snapshots are 132, 116, 104, $105{ }^{\circ} \mathrm{C}$ distribution. Moderate disorientation can explain the fact that the observed valley between the triangles (Fig. 8b) is not deep. From the position of the maximum (a) along the meridian ( $r_{3}$-direction) the average thickness of the nanoscale layers is determined. The lateral extension (c) of the triangles in equatorial ( $r_{12}$-direction) shows the lateral extension of the lamellae. After most of the imperfect domains are molten at $130{ }^{\circ} \mathrm{C}$, we determine a maximum lateral layer extension of $160 \mathrm{~nm}$ from the half base length of the triangular peak.

The drawing on the right-hand side of Fig. 8 shows meridional slices through the CDF as a function of temperature during the heating of the UHMWPE skived film. These curves are known as interface distribution functions (IDF) [35]. They exhibit the size and arrangement of domains in meridional direction. Similar to a tomogram CDF and IDF are computed without making model assumptions on structure. Structure description is following thereafter, based on the features of the image. The arrow in Fig. 8 indicates the long-period depression ("peak") in the IDFs. To the right of the long-period depression no further undulations are visible in the IDF. Such undulations in the IDF would be indicative for cooperative correlation among the crystalline domains. From the missing of correlations it can be concluded that the long period found in the scattering patterns of this material does neither describe a repeat distance in a lattice nor an average repeat in a stack of lamellae. Instead, the nanostructure of the material is dominated by only next-neighbor correlations. An analysis of such a primitive kind of inter-domain correlation has been performed in recent work [16-18], where it was explained by Rényi's random car-parking process [40-43]. In the field of polymer science, already in 1978 Schultz et al. [44] have inferred a predominantly random crystallization mechanism from time-resolved SAXS studies of polyethylene crystallization. Concerning melting of polyethylene, a study of Schultz et al. [45] has indicated a random melt-out (e.g. "de-parking") of crystallites based on SAXS data from isotropic polyethylene. This de-parking is already indicated in the observed extreme broadening of the long period minimum in Fig. 8 at high temperature $\left(130\right.$ and $\left.132{ }^{\circ} \mathrm{C}\right)$.

In order to demonstrate the validity of Schultz's " deparking" mechanism even more clearly, we plot the positions of the first maximum of the IDFs and the positions of the minimum in the IDFs as a function of temperature. In a strongly distorted nanostructure the first parameter value is an approximate value of the average domain thickness ("car-breadth"). The other parameter describes the average distance between two domains ("cars"). The result is shown in Fig. 9. During the heating of the material the most probable thickness of the layers is only slightly increasing from $26 \mathrm{~nm}$ to $30 \mathrm{~nm}$, whereas the 
Fig. 8 Multidimensional CDF with fiber symmetry from BW4 data. UHMWPE during heating at $131{ }^{\circ} \mathrm{C}$. Left: $\mathrm{CDF} z(\mathbf{r})$. The double-arrow indicates the meridional direction. The emphasized curve on the meridian is Ruland's interface distribution function (IDF) [35]. Right: IDFs during heating as a function of temperature. Long periods presented in this work are determined from the positions of the minima (arrow)
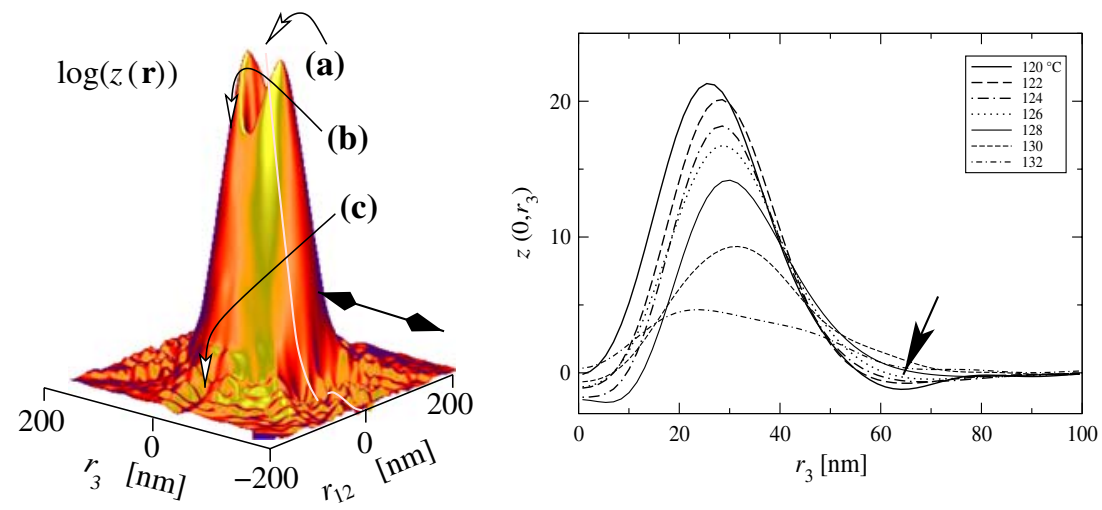

long period is strongly increasing from $60 \mathrm{~nm}$ to $140 \mathrm{~nm}$. Thus, to a first approximation, the melting is described by a process in which the "parking ground" is randomly and continuously emptied from crystallites.

While the original structure exhibits lamellae of up to $160 \mathrm{~nm}$ width, after oriented recrystallization the width of the nanoscale layers has dropped to less than $90 \mathrm{~nm}$, as is derived from the lateral extension of the corresponding peaks in the multidimensional CDFs.

For the data from the anisotropic crystallization experiment the CDFs have been analyzed in the same manner as for the heating process. Figure 10 shows the most probable layer thickness and the long period as a function of crystallization time. During the initial cooling from $136{ }^{\circ} \mathrm{C}$ to $105^{\circ} \mathrm{C}$ spontaneous thickening of the average domain from $14 \mathrm{~nm}$ to $24 \mathrm{~nm}$ is observed. This thickening is accompanied by a decrease of the long period. After the initiation period the average layer thickness is slightly increasing throughout the whole isothermal period, whereas the long period is continuously decreasing during the first $10 \mathrm{~min}$ from $70 \mathrm{~nm}$ to $57 \mathrm{~nm}$. Such a long period

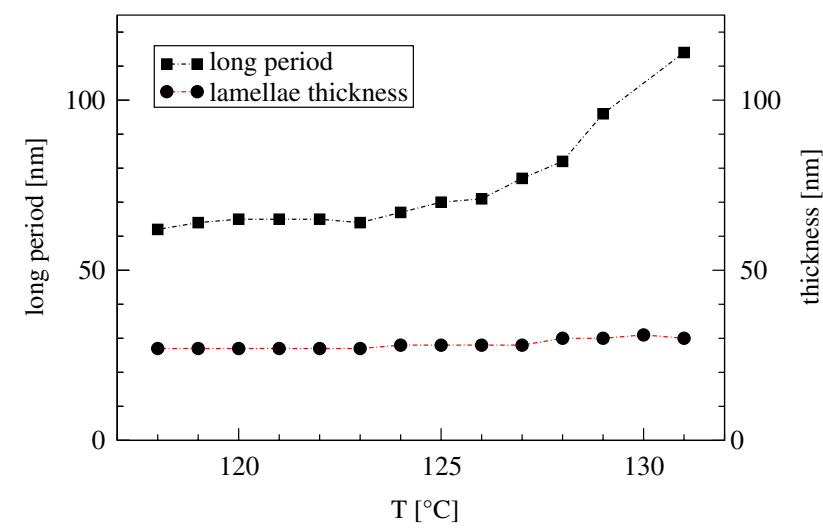

Fig. 9 Heating of UHMWPE. Evolution of the long period and of the most probable layer thickness as extracted from the multidimensional chord distribution function (CDF) decrease is compatible with space filling in a random car parking process [17, 18, 41, 44, 46].

Nine minutes after quenching to the nanoforming temperature a narrow long period peak in the CDF begins to grow broad and high, whereas the early long period is vanishing. Thus a second mechanism of domain arrangement is started after several minutes from a correlation with narrow "grains". With a long-period value of $64 \mathrm{~nm}$ it is very close to the long period of the original skived film. We have no explanation for this process but speculate that such a transition will only be observable in very poorly ordered materials like the one presented in this study.

At room temperature after final quenching a considerable decrease of both layer thickness and long period is observed, as is expected for a semicrystalline nanostructure which is dominated by next-neighbor correlations.

Based on the results of our SAXS experiments carried out at beamline BW4 we have initially set up the hypothesis that the UHMWPE skived film requires a considerable undercooling before crystallization starts. In order to test this hypothesis we have had to carry out combined simul-

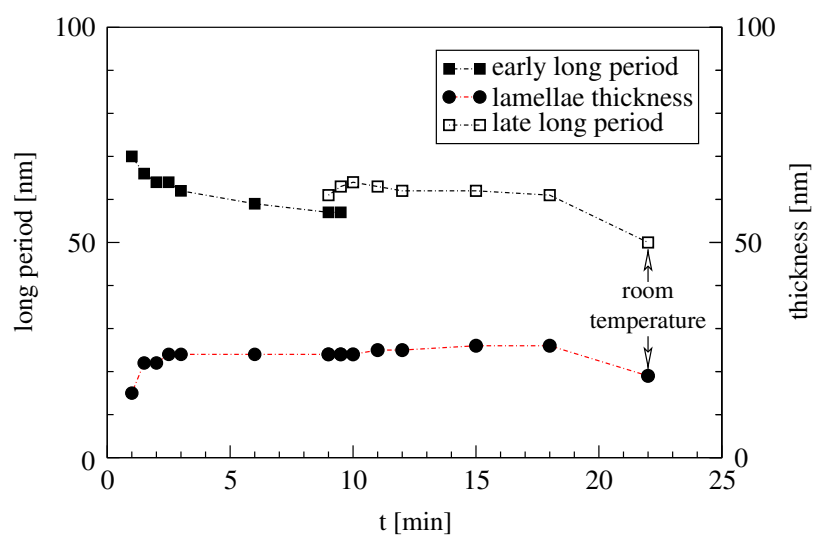

Fig. 10 Isothermal nanoforming of UHMWPE skived film at $105{ }^{\circ} \mathrm{C}$ after melting at $136{ }^{\circ} \mathrm{C}$. Evolution of the long period and of the average "crystalline" layer thickness as extracted from the CDF. The indicated last set of data is from room temperature 

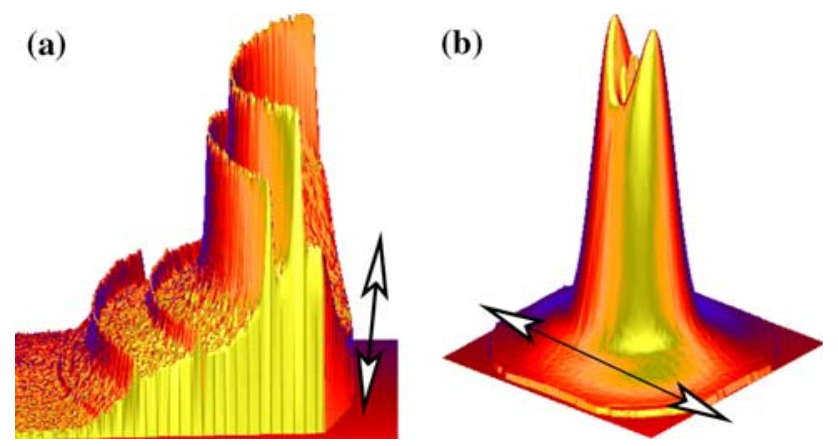

Fig. 11 Combined WAXS/USAXS data of native UHMWPE skived film (Data from ESRF, ID2). The fiber direction is indicated. WAXS intensity is on logarithmic scale. The offset WAXS detector covers an area around the equator of the WAXS pattern. USAXS intensities are on a linear scale. The area of the USAXS patterns covers a range $0.09 \mathrm{~nm}^{-1} \leq s_{12}, s_{3} \leq 0.09 \mathrm{~nm}^{-1}$

taneous 2D WAXS/USAXS measurements with high time resolution (2 $\mathrm{s}$ exposure) during cooling of the material. Such measurements can not be performed at HASYLAB, but at beamline ID02 of ESRF, Grenoble. For the original material before temperature treatment the combined WAXS/USAXS data are presented in Fig. 11. The 2D WAXS pattern is recorded by an offset CCD detector monitoring a region around the equator of the pattern (sample was rotated by $90^{\circ}$ with respect to the usual sample orientation at ID02). The intensity is on a relative logarithmic scale. Obviously the WAXS pattern is isotropic, whereas the USAXS pattern exhibits preferential orientation of lamellae with their planes extending perpendicular to the machine direction (fiber axis) of the skived film.

Figure 12 shows combined WAXS/SAXS data recorded during quenching of the skived film from $136{ }^{\circ} \mathrm{C}$. In the
USAXS patterns from the initiation period of nanostructure formation only an increase of diffuse and isotropic scattering is observed. On the other hand, the WAXS patterns exhibit the main crystalline reflections of polyethylene, as soon as the sample temperature drops to $122{ }^{\circ} \mathrm{C}$. The observed isotropic WAXS reflections demonstrate random orientation of the crystallites.

It appears worth to be noted that the isotropy of only the (110) and the (200) reflections is sufficient to state that the crystallites in the material are oriented at random. This is strictly true, even if we do not have any direct information

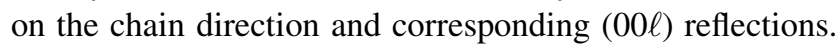
Whenever the normal plane to the chain directions (as defined, e.g., by (110) and (200)) is distributed at random, as well the normal itself must be distributed at random. Only in the case of observed anisotropy the orientations of (110) and (200) reflections are not indicative of the chain orientation.

Despite the random orientation of crystallites (isotropy), anisotropic nanostructure formation is induced by proper choice of the isothermal crystallization temperature. This our observation demonstrates that nanostructure formation in semicrystalline materials may only be loosely coupled to the formation of crystals. First formed in the UHMWPE skived film are small crystals with random orientation and random placement. Second, the formation of more or less oriented imperfect lamellae extending perpendicular to the machine direction of the film can be induced by proper choice of an isothermal nanoforming temperature. According to our results these "lamellae", can be imagined as clusters made from agglomerated and randomly oriented crystallites (Fig. 13) On a higher level these lamellae are correlated to each
Fig. 12 Combined WAXS/ USAXS data of UHMWPE skived film recorded during cooling from a melt-annealing temperature of $136{ }^{\circ} \mathrm{C}$ (Data from ESRF, ID2). The fiber direction is indicated. WAXS intensities are on a relative logarithmic scale. The offset WAXS detector covers an area around the equator of the WAXS pattern. The principal polyethylene crystal reflections are indicated. They are found to be isotropic. USAXS intensities are on an absolute linear scale
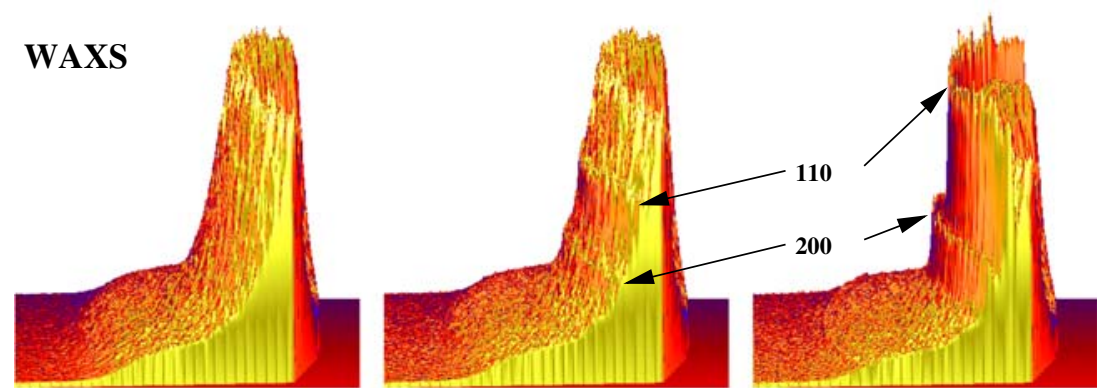

melt $136^{\circ} \mathrm{C}$

$122^{\circ} \mathrm{C}$

$120^{\circ} \mathrm{C}$
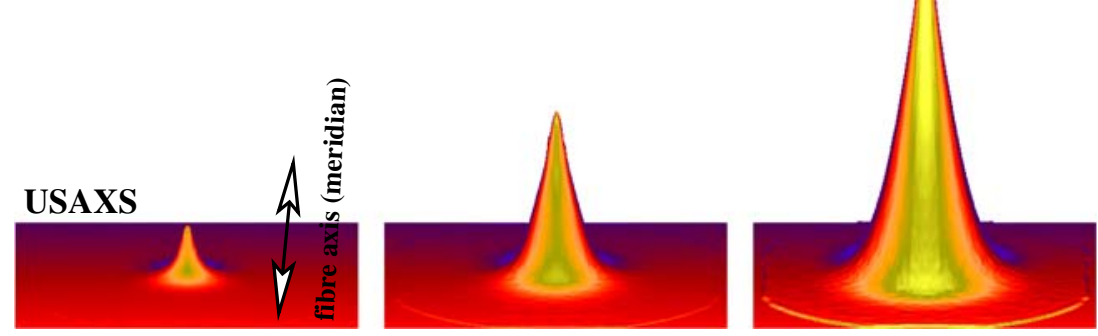


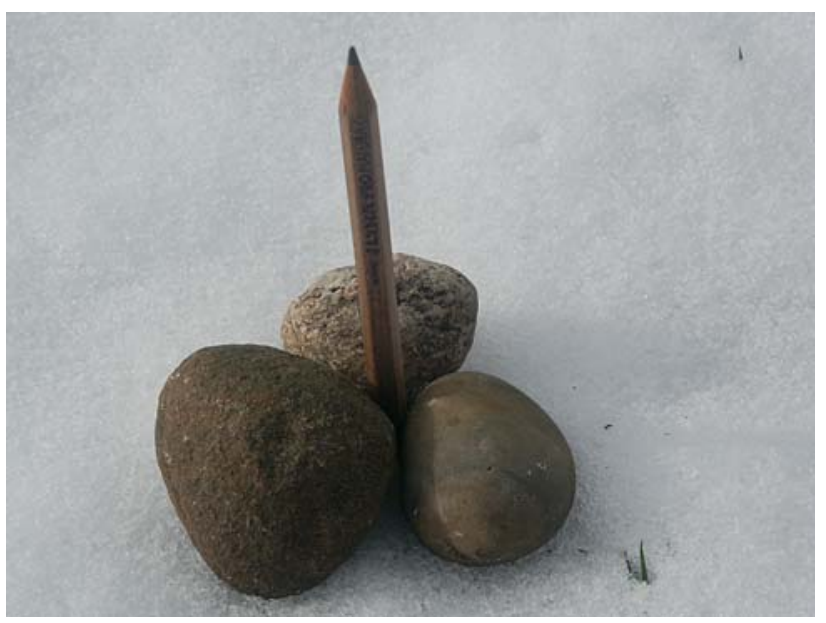

Fig. 13 Model of a layer made by agglomeration in a plane of three differently oriented crystallites (pebbles). The plane is normal to the fiber direction (pencil)

other by only a random sequential volume filling process ("random car parking") [17, 18, 41, 44, 46]. Finally, during quenching, small nanometer-size grains are placed in the center of the remnant amorphous layers. This placement causes the observation of a distinct long period in the final scattering pattern of the processed material (Fig. 1e).

Our results support the notion of Strobl and coworkers [47] that lamellae of polymer nanostructure may be generated by merging of blocky nanodomains. Here the corresponding blocks are clearly identified by their crystalline nature and discriminated from the nanodomain structure by their early formation and random orientation, as can be seen in Fig. 12. Compared to our earlier studies on the oriented crystallization of polyethylene with lower molecular weight the main difference is the fact that a rather high undercooling of the melt is required in order to generate oriented nanodomains and that for the first time a rather slow isothermal nanoforming process has been found that is clearly separated from the preceding fast crystallization process. We speculate that the lateral clustering of randomly oriented crystallites that is already observed with the untreated UHMWPE skived film may have been introduced in the elongation step during the manufacturing of the film.

\section{Conclusions}

Our study shows that in the field of polymer materials research a synchrotron radiation setup for USAXS is a valuable tool to identify interesting processing conditions of nanostructure formation from a polymer melt. On a standard SAXS beamline with a limiting resolution of $50-100 \mathrm{~nm}$ the anisotropy of the pattern would have been overseen. In the first step temperature is changed manually, and the response of the material can immediately be observed. Then, in a second experiment, the evolution of the materials' nanostructure is continuously recorded as a function of processing conditions. For this purpose it is advantageous to have a setup which allows the observation of both 2D-USAXS and 2D-WAXS, as the results of our auxiliary experiment at the ESRF have shown. Such USAXS/WAXS coupling with fast exposure has been an essential precondition to our finding that crystallization and (anisotropic) forming of nanostructure need not be closely related. We admit that the observed nanostructure evolution process is most probably not a general one. Nevertheless it shows that random orientation of polymer crystallites does not necessarily go along with random orientation of lamellae. On the other hand, a layer arrangement according to the simple random car parking process appears to be a frequent mechanism in polymer two-phase materials with short-range domain order.

Acknowledgements We acknowledge HASYLAB, Hamburg, for provision of the synchrotron radiation facilities at beamline BW4 in the frame of project II-04-039. In particular the support of the beamline engineers M. Dommach and R. Döhrmann is greatly appreciated. ESRF, Grenoble is acknowledged for provision of synchrotron radiation facilities at beamline ID02 in the frame of project SC-1679. Financial support of this study by the Deutsche Forschungsgemeinschaft (DFG STR501/4-1) is gratefully acknowledged.

\section{References}

1. Heck B, Hugel T, Iijima M, Sadiku E, Strobl G (1999) New J Phys $1: 17.1$

2. Heck B, Hugel T, Iijima M, Strobl G (2000) Polymer 41:8839

3. Heeley EL, Maidens AV, Olmsted PD, Bras W, Dolbnya IP, Fairclough JPA, Terrill NJ, Ryan AJ (2003) Macromolecules 36:3656

4. Bras W, Dolbnya I, Detollenaere D, van Tol R, Malfois M, Greaves G, Ryan A, Heeley E (2003) J Appl Cryst 36:791

5. Somani RH, Yang L, Hsiao BH, Fruitwala H (2003) J Macromol Sci Part B Phys B42:515

6. Somani RH, Yang L, Hsiao BS, Agarwal PK, Fruitwala HA, Tsou AH (2002) Macromolecules 35:9096

7. Yamazaki S, Hikosaka M, Toda A, Wataoka I, Yamada K, Tagashira K (2003) J Macromol Sci Part B: Phys B42:499

8. Allegra G, Meille SV (1999) Phys Chem Chem Phys 1:5179

9. Pearce R, Vancso GJ (1998) Polymer 39:1237

10. Hobbs JK, Humphris ADL, Miles MJ (2001) Macromolecules 34:5508

11. Humphris ADL, Hobbs JK, Miles MJ (2003) Appl Phys Lett 83:6

12. Stribeck N (1993) Colloid Polym Sci 271:1007

13. Stribeck N (2000) ACS Symp Ser 739:41

14. Stribeck N (2001) J Appl Cryst 34:496

15. Stribeck N (2002) Colloid Polym Sci 280:254

16. Stribeck N, Almendarez Camarillo A, Cunis S, Bayer RK, Gehrke R (2004) Macromol Chem Phys 205:1445

17. Stribeck N (2004) Macromol Chem Phys 205:1455

18. Stribeck N, Almendarez Camarillo A, Bayer R (2004) Macromol Chem Phys 205:1463 
19. Stribeck N, Bayer R, Bösecke P, Almendarez Camarillo A (2005) Polymer 46:2579

20. Stribeck N, Bösecke P, Bayer R, Almendarez Camarillo A (2005) Progr Coll Polym Sci 130:127

21. Bösecke P, Diat O (1997) J Appl Cryst 30:867

22. VNI "pv-wave manuals" V 7.5 (2001), Boulder, Colorado

23. Bonart R (1966) Kolloid Z u Z Polymere 211:14

24. Fischer EW (1969) Colloid Polym Sci 231:458

25. Buhmann MD (2000) Acta Num 9:1

26. Stribeck N (2003) Anal Bioanal Chem 376:608

27. Stribeck N, Buzdugan E, Ghioca P, Serban S, Gehrke R (2002) Macromol Chem Phys 203:636

28. Stribeck N, Bayer R, von Krosigk G, Gehrke R (2002) Polymer 43:3779

29. Stribeck N (2003) Fibr Text EE 11:33

30. Stribeck N, Androsch R, Funari SS (2003) Macromol Chem Phys 204:1202

31. Stribeck N, Fakirov S, Apostolov AA, Denchev Z, Gehrke R (2003) Macromol Chem Phys 204:1000
32. Stribeck N, Funari SS (2003) J Polym Sci Part B: Polym Phys 41:1947

33. Stribeck N, Fakirov S (2001) Macromolecules 34:7758

34. Vonk CG (1979) Colloid Polym Sci 257:1021

35. Ruland W (1977) Colloid Polym Sci 255:417

36. Ruland W (1978) Colloid Polym Sci 256:932

37. Stribeck N, Ruland W (1978) J Appl Cryst 11:535

38. Porod G (1972) Monatsh Chem 103:395

39. Cohen Y, Thomas EL (1987) J Polym Sci, Part B: Polym Phys B25:1607

40. Rényi A (1958) Publ Math Inst Budapest 3:109

41. Rényi A (1963) Sel Transl Math Stat Prob 4:203

42. Burgos E, Bonadeo H (1987) J Phys A 20:1193

43. Bonnier B, Boyer D, Viot P (1994) J Phys A 27:3671

44. Schultz JM, Lin JS, Hendricks RW (1978) J Appl Cryst 11:551

45. Schultz JM, Fischer EW, Schaumburg O, Zachmann HG (1980) J Polym Sci Polym Phys 18:239

46. Evans JW (1993) Rev Mod Phys 65:128

47. Hugel T, Strobl G, Thomann R (1999) Acta Polym 50:214 